\title{
Circulating microRNAs as novel biomarkers for dilated cardiomyopathy
}

\author{
Hua Wang ${ }^{1 *}$, Feng Chen ${ }^{2 *}$, Jiabin Tong ${ }^{1}$, Yingying $\mathrm{Li}^{1}$, Jianping Cai ${ }^{3}$, Yan Wang ${ }^{1}$, \\ Peng $\mathrm{Li}^{1}$, Yichun $\mathrm{Hao}^{1}$, Weimeng Tian ${ }^{1}$, You Lv ${ }^{1}$, Jia Chong ${ }^{1}$, Jiefu Yang ${ }^{1}$
}

${ }^{1}$ Department of Cardiology, Beijing Hospital, National Center of Gerontology, 100730, Beijing, China ${ }^{2}$ Department of Cardiology, Fuwai Hospital, National Center for Cardiovascular Diseases, Chinese Academy of Medical Sciences and Peking Union Medical College, 100037, Beijing, China

${ }^{3}$ The Key Laboratory of Geriatrics, Beijing Hospital, National Center of Gerontology, Beijing, 100730, China

\begin{abstract}
Background: Circulating microRNAs (miRNAs) potentially carry disease-specific information. In the current study, we aim to characterize the miRNA signature in plasma from dilated cardiomyopathy (DCM) patients and assess the possible correlation between expression levels of circulating miRNAs and symptom severity in DCM patients.

Methods: Using microarray-based miRNA expression profiling, we compared the miRNA expression levels in plasma samples from 4 DCM patients and 3 healthy controls. The expression levels of selected differentially expressed, upregulated miRNAs (miR-3135b, miR-3908 and miR-5571-5p) were validated independently in plasma samples from 19 DCM patients and 20 controls.

Results: We observed that plasma miR-3135b ( $p<0.001)$, miR-3908 $(p<0.001)$ and miR-5571-5p $(p<0.001)$ were significantly upregulated in DCM patients. The area under receiver operating characteristic (ROC) curves for the 3 miRNAs ranged from 0.83 to 1.00. Moreover, miR-5571-5p levels in plasma were significantly upregulated with severe New York Heart Association (NYHA) classification $(p<0.05)$.

Conclusions: The circulating miRNAs (miR-3135b, miR-3908 and miR-5571-5p) have potential as diagnostic biomarkers for DCM. Additionally, miR-5571-5p correlated with NYHA classification. (Cardiol J 2017; 24, 1: 65-73)
\end{abstract}

Key words: microRNAs, dilated cardiomyopathy, biomarkers, diagnosis

\section{Introduction}

Dilated cardiomyopathy (DCM), which is generally used as an all-encompassing term for a non-ischemic cardiomyopathy with depressed left ventricular (LV) function, is an important cause of sudden cardiac death and heart failure (HF) and also the leading indication for cardiac transplantation in children and adults worldwide [1]. DCM may manifest clinically at a wide range of ages and is often identified when associated with severe limiting symptoms and disability. The diagnosis of DCM is usually made with echocardiography, which has several disadvantages, including high financial costs, limited availability and high interobserver variability. The use of circulating biomarkers in the diagnostic approach to DCM would offer an attractive alternative. MicroRNAs (miRNAs) are small, highly conserved, non-coding RNAs, which act as endogenous inhibitors of target genes [2-5]. MiRNAs have been shown to be key molecular players in nearly all cellular processes, including

Address for correspondence: Dr. Jiefu Yang, Department of Cardiology, Beijing Hospital, National Center of Gerontology, 100730, Beijing, China, tel: +86 13601292259, e-mail: yangjiefu2011@126.com

*These authors contributed equally to this work and should be considered co-first authors.

Received: 20.03.2016 Accepted: 06.10.2016 
cardiovascular development and pathophysiology $[6,7]$. Gain or loss of function experiments, expression arrays and bioinformatic analysis confirmed that specific miRNAs played essential roles in the biogenesis of DCM [6, 8-13]. This observation laid the theoretical foundation of the diagnostic application of miRNAs to DCM. Subsequently, several studies demonstrated miRNA expression differences in various pathological conditions and characterized the links between specific miRNAs and aspects of pathologies $[14,15]$. In addition, circulating miRNAs have many requisite features of good biomarkers, such as accessibility through noninvasive methods; a high degree of specificity and sensitivity; the ability to differentiate pathologies, allowing early detection; sensitivity to relevant changes in the disease; a long half-life within the sample; and the capability for rapid and accurate detection [16]. Therefore, circulating miRNAs have potential as new diagnostic biomarkers for DCM.

In the present study, we aimed to characterize the miRNA signature in plasma from DCM patients compared with healthy controls. We also evaluated the discriminatory power of the significantly upregulated miRNAs and correlated them with symptom severity in DCM.

\section{Methods}

\section{Patients and controls cohort}

This study has been approved by the Institution Review Board of Beijing Hospital of the Ministry of Health, and all subjects provided written informed consent before the initiation of any study-related procedures. Twenty-three patients with DCM with no identifiable or reversible etiology were recruited from the Department of Cardiology. Subjects that demonstrated persistent ( $>3$ months) LV systolic dysfunction (LV ejection fraction $[\mathrm{LVEF}] \leq 40 \%$ ) as assessed by echocardiography were classified as patients with DCM for the study. Each DCM patient underwent coronary computed tomography or coronary angiography, which revealed that no coronary stenosis was larger than $50 \%$. In addition, a control group, consisting of 23 apparently healthy volunteers with no known heart disease, kidney, lung or hematologic disorders, were recruited; they were age- and gender-matched to the DCM group. Exclusion criteria for all participants were pregnancy, known or treated malignancies, and connective diseases.

All of the clinically relevant data were collected for the DCM group and control group: clinical, echocardiographic, electrocardiographic, and baseline laboratory, as well as the relevant demographic data.

\section{Sample acquisition and handling}

An $8 \mathrm{~mL}$ aliquot of blood was obtained from all participants directly into sodium citrate tubes. The whole blood was allowed to stand for $\sim 3 \mathrm{~h}$ at $-4^{\circ} \mathrm{C}$ before centrifuging at $1,500 \mathrm{~g}$ for $10 \mathrm{~min}$ at room temperature. The resultant plasma was aliquoted into Eppendorf tubes and stored at $-70^{\circ} \mathrm{C}$.

\section{Clinical biochemical markers}

Peripheral venous blood samples were tested for the levels of hemoglobin (HGB), creatinine, aspartate aminotransferase (AST), and alanine aminotransferase (ALT) using standard protocols in the core clinical laboratory at Peking University Fifth Hospital. Plasma N-terminal-pro-B type natriuretic peptide (NT-proBNP) was measured by electrochemiluminescence immunoassay (Elecsys proBNP II assay) on an automated Cobas e601 analyzer according to the manufacturer's instructions (Roche Diagnostics GmbH, Mannheim, German). The quality controls were measured within 2 -standard deviation values. The range of detection for NT-proBNP was $5-3,5000 \mathrm{pg} / \mathrm{mL}$.

\section{Echocardiography}

Doppler echocardiographic assessment was performed by one of 2 blinded operators in accordance with the European Society of Echocardiography recommendations. All data represent the mean of 3 measurements on sequential cardiac cycles. LVEF was calculated by the Simpson's method.

\section{RNA extraction}

Total RNA was isolated using TRIzol (Invitrogen) and purified with RNeasy mini kit (QIAGEN) according to manufacturer's instructions. RNA quality and quantity was measured by using a nanodrop spectrophotometer (ND-1000, Nanodrop Technologies), and RNA Integrity was determined by gel electrophoresis.

\section{Microarray processing and analysis}

RNA labeling and array hybridization was performed according to Exiqon's manual. The samples were labeled using the miRCURY ${ }^{\mathrm{TM}} \mathrm{Hy} 3^{\mathrm{TM}} / \mathrm{Hy} 5^{\mathrm{TM}}$ Power labeling kit (Exiqon, Vedbaek, Denmark) and hybridized on the miRCURY ${ }^{\mathrm{TM}}$ LNA Array (v.18.0) (Exiqon) according to the manufacturer's guidelines. Following hybridization, the slides were obtained and washed several times using Wash buffer kit (Exiqon). Then, the slides were 
scanned using the Axon GenePix 4000B microarray scanner (Axon Instruments, Foster City, CA). Scanned images were then imported into GenePix Pro 6.0 software (Axon) for grid alignment and data extraction. Replicated miRNAs were averaged and miRNAs with intensities $\geq 30$ in all samples were chosen for calculating the normalization factor. Expression data were normalized using the median normalization. After normalization, the miRNAs were considered to be significantly differentially expressed only when they exhibited a mean change $>2$-fold relative to the controls and had a q-value $<0.05$ between the DCM patients and control groups.

\section{Quantitative reverse-transcription} polymerase chain reaction (qRT-PCR)

To confirm the findings obtained by analyzing the miRNA profiling, we measured the expression of upregulated miRNAs using TaqMan qRT-PCR. All reactions were carried out using Gene Amp PCR System 9700 (Applied Biosystems), according to the manufacturer's recommendations. In brief, the reverse transcription reaction was performed in $20 \mu \mathrm{L}$ mixture consisting of $0.3 \mu \mathrm{L}$ of $10 \mu \mathrm{M}$ RT primer, and $100 \mathrm{ng}$ RNA sample, $2 \mu \mathrm{L}$ of $2.5 \mathrm{mM}$ dNTPs, $0.2 \mu \mathrm{L}$ MMLV reverse transcriptase, $2 \mu \mathrm{L}$ of $10 \times$ reverse transcription buffer, $0.3 \mu \mathrm{L}$ RNase inhibitor, and nuclease-free water. The reaction mixture was incubated for $30 \mathrm{~min}$ in $16^{\circ} \mathrm{C}, 40 \mathrm{~min}$ in $42^{\circ} \mathrm{C}$, and $5 \mathrm{~min}$ in $85^{\circ} \mathrm{C}$, and then kept in $-20^{\circ} \mathrm{C}$. Next, qPCR was performed in a final volume of $10 \mu \mathrm{L}$ containing $1 \mu \mathrm{L}$ PCR probe, $2 \mu \mathrm{L}$ product from the RT reaction, $5 \mu \mathrm{L} 2 \mathrm{X}$ PCR Master Mix, and $2 \mu \mathrm{L}$ nuclease-free water. The thermal cycle started with $10 \mathrm{~min}$ at $95^{\circ} \mathrm{C}$, followed by 40 cycles of $95^{\circ} \mathrm{C}$ for $10 \mathrm{~s}$ and $60^{\circ} \mathrm{C}$ for $1 \mathrm{~min}$. MiR-191-5p was selected as the internal control miRNA to normalize the cDNA levels of different samples because the concentration of miR-191-5p was approximately the same in various samples (detailed data can be found in Table 1). The threshold cycle $(\mathrm{Ct})$ values obtained for each miRNA were then normalized to the respective miR-191-5p Ct values to obtain the $\Delta \mathrm{Ct}$ values and eventually used to plot relative expression values.

\section{Data analysis and statistics}

Data are presented as mean \pm standard deviation unless indicated otherwise, and plasma miRNA levels are presented as fold change relative to controls. The miRNA expression fold changes were calculated using the arithmetic formula $2^{-\Delta \Delta \mathrm{CT}}$.

Continuous clinical variables were compared between the two groups by a 2 -sided unpaired
Table 1. MiR-191-5p expression of all samples. Coefficient of variation: $1.1 \%$. Circulating miR$-191-5 p$ is stably expressed across all samples and thus is a suitable reference gene for normalizing reverse-transcription polymerase chain reaction data in this study.

\begin{tabular}{|c|c|}
\hline Sample & (miR-191-5p) threshold cycle \\
\hline Control1 & 22.071 \\
\hline Control2 & 22.469 \\
\hline Control3 & 22.278 \\
\hline Control4 & 21.988 \\
\hline Control5 & 22.113 \\
\hline Control6 & 21.469 \\
\hline Control7 & 22.111 \\
\hline Control8 & 21.609 \\
\hline Control9 & 21.933 \\
\hline Control10 & 21.678 \\
\hline Control11 & 21.711 \\
\hline Control12 & 21.849 \\
\hline Control13 & 22.078 \\
\hline Control14 & 21.906 \\
\hline Control15 & 21.893 \\
\hline Control16 & 22.060 \\
\hline Control17 & 21.548 \\
\hline Control18 & 21.946 \\
\hline Control19 & 21.895 \\
\hline Control20 & 21.585 \\
\hline Dilated1 & 21.821 \\
\hline Dilated2 & 21.977 \\
\hline Dilated3 & 22.007 \\
\hline Dilated4 & 21.603 \\
\hline Dilated5 & 21.714 \\
\hline Dilated6 & 21.644 \\
\hline Dilated7 & 22.083 \\
\hline Dilated8 & 21.887 \\
\hline Dilated9 & 21.988 \\
\hline Dilated 10 & 22.237 \\
\hline Dilated11 & 22.042 \\
\hline Dilated12 & 21.677 \\
\hline Dilated13 & 21.491 \\
\hline Dilated14 & 21.465 \\
\hline Dilated15 & 21.536 \\
\hline Dilated16 & 22.153 \\
\hline Dilated17 & 22.372 \\
\hline Dilated18 & 22.075 \\
\hline Dilated19 & 21.943 \\
\hline
\end{tabular}

t-test for Gaussian data or Mann-Whitney tests for non-Gaussian data. Chi-square tests were used to compare categorical clinical variables. 
Table 2. Characteristics of the dilated cardiomyopathy (DCM) group and the control group.

\begin{tabular}{|c|c|c|c|c|}
\hline \multirow[t]{2}{*}{ Variables } & \multicolumn{2}{|c|}{ Cohort 1 (screening by microarray) } & \multicolumn{2}{|c|}{ Cohort 2 (validation by qRT-PCR) } \\
\hline & $\begin{array}{c}\text { Controls } \\
(n=3)\end{array}$ & $\begin{array}{c}\text { DCM } \\
(n=4)\end{array}$ & $\begin{array}{l}\text { Controls } \\
(n=20)\end{array}$ & $\begin{array}{c}\text { DCM } \\
(n=19)\end{array}$ \\
\hline Age [years] & $49.3 \pm 8.4$ & $53.7 \pm 7.6$ & $56.7 \pm 9.1$ & $59.2 \pm 13.1$ \\
\hline Male & $2(66.7 \%)$ & $4(100 \%)$ & $16(80 \%)$ & $16(84.2 \%)$ \\
\hline Body mass index $\left[\mathrm{kg} / \mathrm{m}^{2}\right]$ & $27.2 \pm 0.7$ & $25.4 \pm 2.7$ & $25.4 \pm 2.1$ & $27.0 \pm 5.0$ \\
\hline Diabetes & $0(0 \%)$ & $0(0 \%)$ & $0(0 \%)$ & $2(10.5 \%)$ \\
\hline Hypertension & $0(0 \%)$ & $2(50 \%)$ & $0(0 \%)$ & $12(63.2 \%)^{\wedge}$ \\
\hline Coronary artery disease & $0(0 \%)$ & $0(0 \%)$ & $0(0 \%)$ & $0(0 \%)$ \\
\hline Atrial fibrillation & $0(0 \%)$ & $0(0 \%)$ & $0(0 \%)$ & $9(47.4 \%)^{\wedge}$ \\
\hline NT-proBNP [pg/mL] & $29.7(5.9-51.4)$ & $1352.8(772.0-18050.0)^{\#}$ & $39.2(13.4-62.1)$ & $1991.9(851.5-3706.0)^{\wedge}$ \\
\hline LVEF [\%] & $68.3 \pm 2.1$ & $20.5 \pm 3.1^{\wedge}$ & $65.8 \pm 4.9$ & $27.5 \pm 7.7^{\wedge}$ \\
\hline LAD [mm] & $31.0 \pm 2.6$ & $47.3 \pm 5.7^{\wedge}$ & $34.8 \pm 4.1$ & $45.4 \pm 6.5^{\wedge}$ \\
\hline LVEDD [mm] & $44.3 \pm 2.3$ & $71.0 \pm 9.1^{\wedge}$ & $45.1 \pm 3.3$ & $61.4 \pm 7.1^{\wedge}$ \\
\hline \multicolumn{5}{|l|}{ NYHA grade: } \\
\hline Class I/II & & $2(50 \%)$ & & $6(31.5 \%)$ \\
\hline Class III/IV & & $2(50 \%)$ & & $13(68.5 \%)$ \\
\hline Hemoglobin [g/L] & $153.7 \pm 27.5$ & $152.8 \pm 14.1$ & $153.3 \pm 17.2$ & $149.7 \pm 19.1$ \\
\hline eGFR [mL/min] & $116.9 \pm 28.5$ & $80.7 \pm 11.4$ & $105.5 \pm 22.2$ & $91.1 \pm 34.9$ \\
\hline ALT [U/L] & $14.3 \pm 1.2$ & $22.0 \pm 13.0$ & $29.2 \pm 29.2$ & $19.9 \pm 9.3$ \\
\hline AST [U/L] & $19.0 \pm 5.6$ & $25.8 \pm 7.2$ & $28.3 \pm 11.0$ & $23.8 \pm 4.6$ \\
\hline Beta-blockers & $0(0 \%)$ & $4(100 \%)^{\#}$ & $0(0 \%)$ & $18(94.7 \%)^{\wedge}$ \\
\hline ACEI/ARB & $0(0 \%)$ & $4(100 \%)^{\#}$ & $0(0 \%)$ & $18(94.7 \%)^{\wedge}$ \\
\hline Spironolactone & $0(0 \%)$ & $4(100 \%)^{\#}$ & $0(0 \%)$ & $17(89.5 \%)^{\wedge}$ \\
\hline Digitalis & $0(0 \%)$ & $3(75 \%)$ & $0(0 \%)$ & $4(21.1 \%)^{\#}$ \\
\hline Antiplatelet & $0(0 \%)$ & $0(0 \%)$ & $0(0 \%)$ & $0(0 \%)$ \\
\hline Anticoagulation & $0(0 \%)$ & $0(0 \%)$ & $0(0 \%)$ & $9(47.4 \%)^{\wedge}$ \\
\hline ICD/CRT & $0(0 \%)$ & $1(25 \%)$ & $0(0 \%)$ & $13(68.4 \%)^{\wedge}$ \\
\hline
\end{tabular}

Values are shown as mean \pm standard deviation, $\mathrm{n}(\%)$, or median (interquartile range); $\mathrm{p}<0.05 ; \# \mathrm{p}<0.01 ; \mathrm{qRT}-\mathrm{PCR}-\mathrm{quantitative}$ reverse-transcription polymerase chain reaction; NT-proBNP - N-terminal-pro-B type natriuretic peptide; LVEF — left ventricular ejection fraction; LAD — left atrial dimension; LVEDD — left ventricular end-diastolic dimension; NYHA — New York Heart Association; eGFR - estimated glomerular filtration rate; ALT — alanine aminotransferase; AST — aspartate aminotransferase; ACEI — angiotensin-converting enzyme inhibitor; ARB — angiotensin receptor blockers; ICD — implantable cardioverter-defibrillator; CRT — cardiac resynchronization therapy

Receiver operating characteristic (ROC) analysis was carried out for each miRNA validated by qRT-PCR, as well as NT-proBNP, and the area under the ROC curve (AUC) was calculated to evaluate its predictive power for DCM.

Associations between the New York Heart Association (NYHA) classification and miRNAs were evaluated as follows: a 2 -sided unpaired t-test was used to compare the miRNAs of samples with NYHA classifications of class I-II with the samples with NYHA classifications of class III-IV.

Data analysis was performed using the Statistical Package for the Social Sciences (version 19.0; SPSS Inc., Chicago, IL, USA). The probability level for statistical significance was set at $\alpha=0.05$.

\section{Results}

\section{Patient characteristics}

To select candidate plasma miRNAs for DCM detection, we performed an initial genome-wide microarray screening of Cohort 1 : samples derived form $4 \mathrm{DCM}$ patients and 3 matched controls. Then, we studied Cohort 2, which was composed of $19 \mathrm{DCM}$ patients and 20 controls for independent validation. The clinical characteristics of the DCM patients and controls are shown in Table 2. In both cohorts, the two groups were similar regarding age, gender, body mass index, HGB, liver function (ALT, AST) and renal function (estimated glomerular filtration rate [eGFR]). Predicted 
significant changes, reflecting the differences between DCM patients vs. controls were in LVEF, left atrial dimension, LV end-diastolic dimension and NT-proBNP. In Cohort 2, DCM patients had significantly higher levels of NT-proBNP: $\sim 1,991.9 \mathrm{pg} / \mathrm{mL}$ compared with controls: $\sim 39.2$ $\mathrm{pg} / \mathrm{mL}$. The LVEF in DCM patients was $\sim 27.5 \%$ compared with controls with a LVEF of $\sim 65.8 \%$.

\section{MiRNAs microarray of plasma in DCM}

To determine the differential miRNA expression in DCM, we performed plasma miRNA microarray analysis with $4 \mathrm{DCM}$ patients and 3 controls. The microarray covered all human, mouse and rat miRNAs annotated in miRBase 18.0, as well as all viral miRNAs related to these species. The expression profiles of altered miRNAs following the genome-wide miRNA microarray showed distinctive differences between control and DCM patient sample. Of 3,100 miRNAs detected on the microarray, 47 miRNAs were found to be differentially expressed in DCM patients compared with controls with a fold change $>2$ and $q<0.05$, among which 30 miRNAs were increased. Especially miR-3135b, miR-3908, and miR-5571-5p had the most significantly increased level.

In some stances, studies have reported that the peripheral blood mononuclear cells had similar expression of certain gene expression compared to pathological tissues. To test whether differentially expressed miRNAs of plasma from DCM patients mimic the pattern of cardiac miRNAs in end-stage HF because of DCM, 9 miRNAs representing the cardiac miRNA signature were assessed [10]. Surprisingly, we observed that 3 miRNAs (miR-1, miR-342, and miR-145) were barely detectable in the microarray and that none of the 6 remaining miRNAs significantly mirror the cardiac miRNA expression pattern (Fig. 1). This indicates that circulating miRNA in plasma may be different and might have a unique miRNA profile.

\section{Quantitative RT-PCR analysis of plasma miRNAs in DCM}

Within Cohort 1, miR-3135b, miR-3908, and miR-5571-5p had been shown to have significantly upregulated expression. To verify the differential expression patterns of these miRNAs, we further examined these 3 miRNAs in independent Cohort 2 , which consisted of $19 \mathrm{DCM}$ patients and 20 controls, by qRT-PCR. Consistent with the aforementioned results, miR-3135b ( $<<0.001)$, miR-3908 ( $\mathrm{p}<0.001)$, and miR-5571-5p $(\mathrm{p}<0.001)$ were significantly upregulated in DCM patients com-

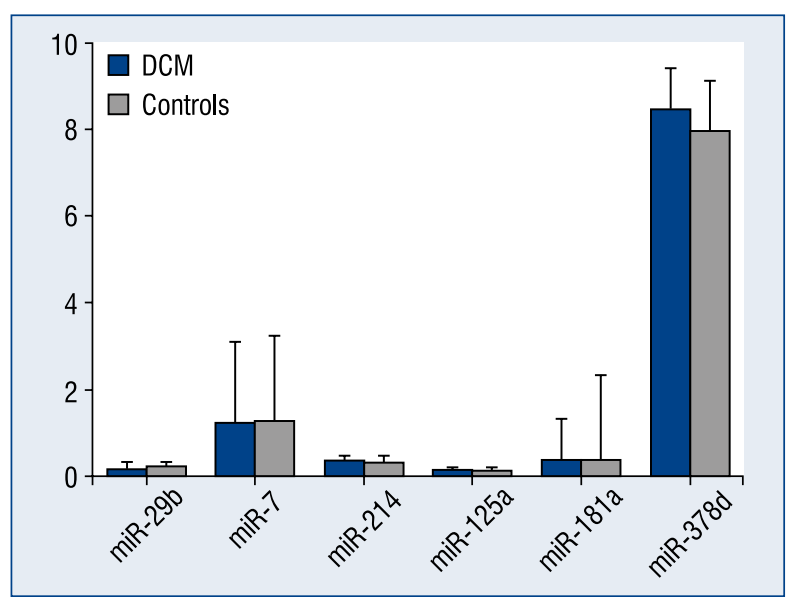

Figure 1. MiRNA microarray analysis showed that 6 miRNAs representing the cardiac miRNA signature of dilated cardiomyopathy (DCM) patients were not significantly differentially expressed in plasma from DCM patients compared with plasma from controls. Data presented as the mean \pm standard deviation.

pared with controls (Fig. 2). Furthermore, our studies revealed that the miR-3135b, miR-3908, and miR-5571-5p expression levels were even altered in plasma from patients who had just been classified into NYHA class I/II, suggesting that plasma miRNAs changes could be observed even in patients with mild symptoms.

Furthermore, ROC analysis showed miR-3135b, miR-3908, and miR-5571-5p with significant AUC ranged from 0.83 to 1.00 , implying that they had the discriminatory power to distinguish DCM patients from controls (Fig. 3). For comparison with the conventional biomarker of HF, we used NT-proBNP measurements from these patients and NT-proBNP exhibited an AUC value of 0.99 . Thus, miR-3135b and miR-5571-5p had a similar discriminative power for DCM with NT-proBNP.

Because miRNAs are normally excreted by the kidney [17], it is possible that miRNA levels in the plasma are affected by renal function. To assess whether such an effect may have caused a bias in our study, we compared the miRNA levels between DCM patients whose values for the eGFR were above the median and the samples with values that were below the median. There was no significant $(\mathrm{p}<0.05)$ difference for each miRNA.

\section{Correlation between miRNAs and symptom severity in DCM}

The 3 miRNAs were tested for a possible association with symptom severity in DCM. NYHA 


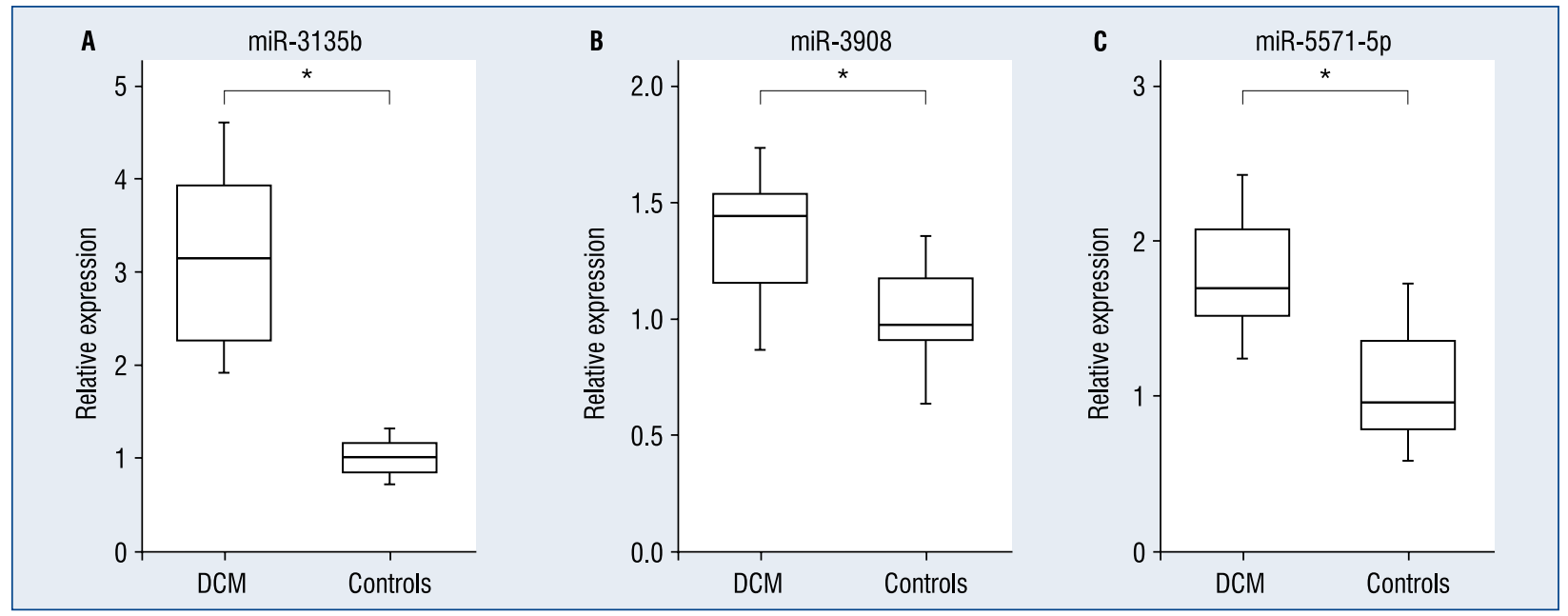

Figure 2. Levels of miR-3135b (A), miR-3908 (B), and miR-5571-5p (C) in dilated cardiomyopathy (DCM) patients compared with controls by reverse-transcription polymerase chain reaction. Data are presented as fold over internal control -miR-191-5p; ${ }^{*} p<0.05$.

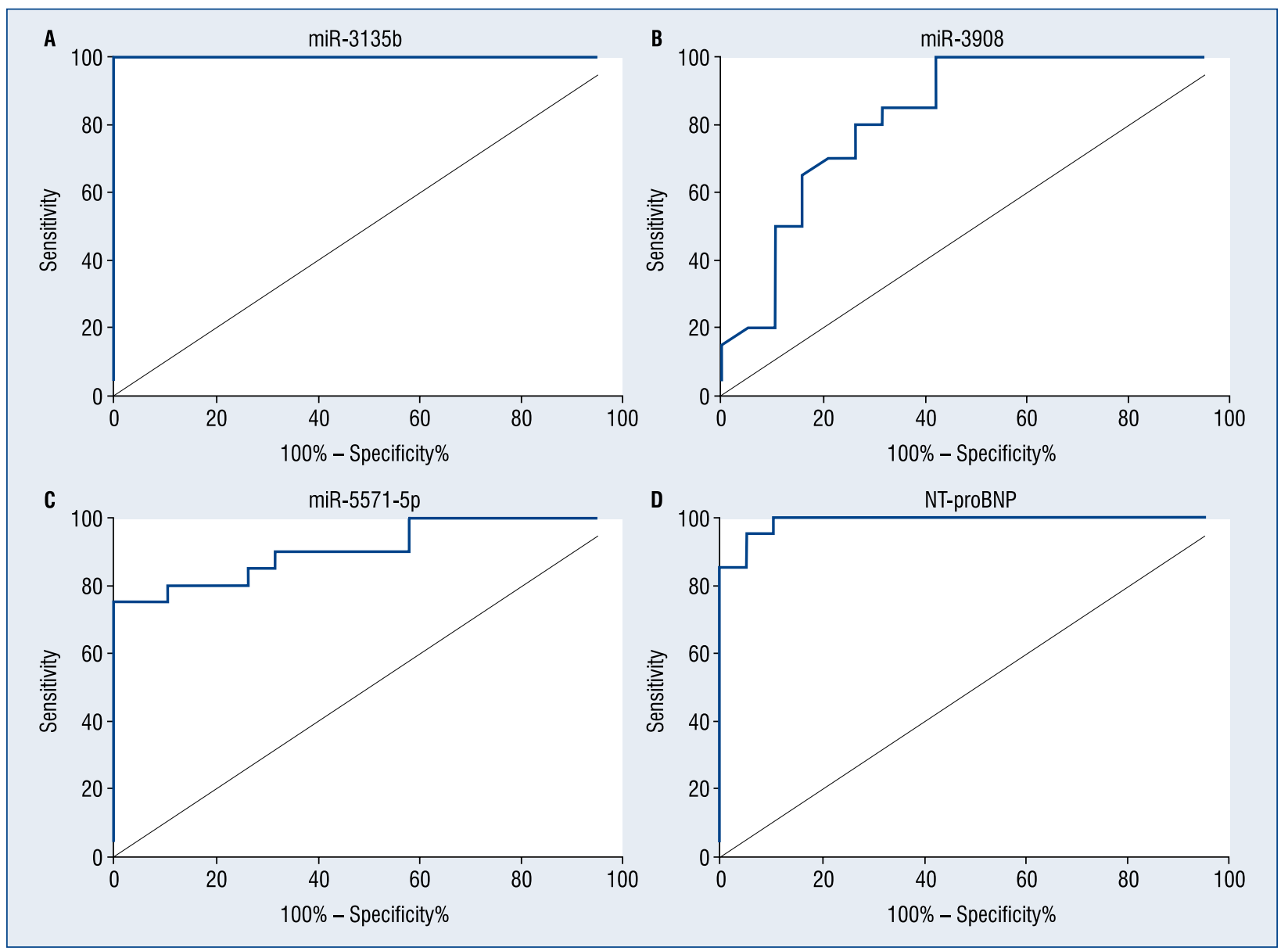

Figure 3. Receiver operating characteristic (ROC) analysis. ROC curves for miR-3135b (A), miR-3908 (B), and miR-5571-5p (C) showed the area under the ROC curve (AUC) to be 1.00, 0.83 (95\% Cl 0.70-0.96), and 0.91 (95\% Cl 0.82-1.00), respectively. Additionally, N-terminal-pro-B type natriuretic peptide (NT-proBNP) values were used for ROC analysis, and the AUC was 0.99 (95\% Cl 0.97-1.00) (D). 


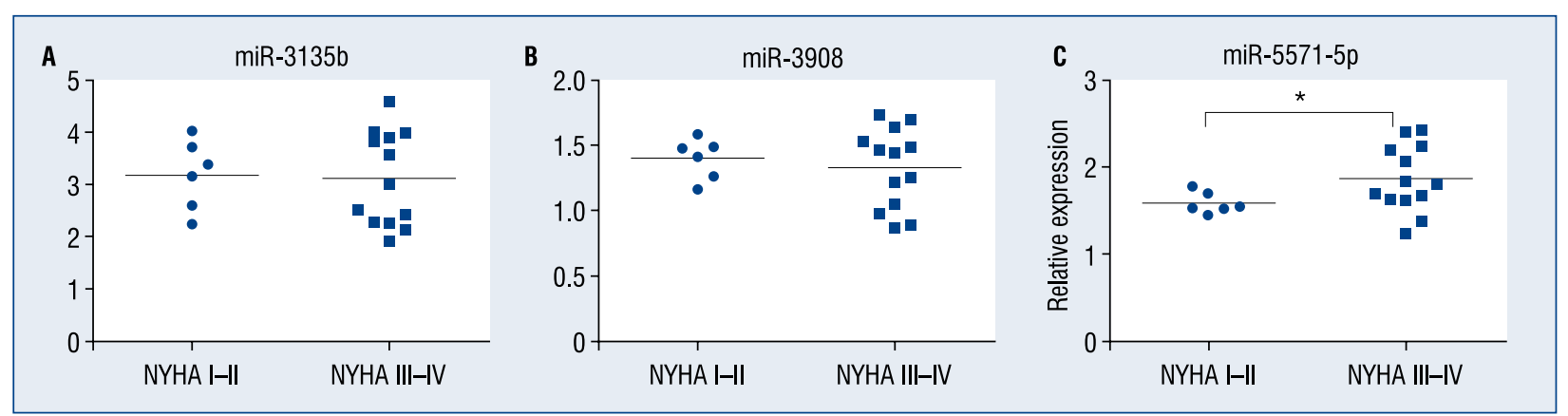

Figure 4. Significant associations between symptom severity and individual microRNA. There are no significant associations between New York Heart Association (NYHA) and miR-3135b (A) or miR-3908 (B). Additionally, more severe NYHA functional classification is associated with higher miR-5571-5p levels $(\mathbf{C})$; ${ }^{*} p<0.05$.

classification was based on the severity of symptoms and physical activity. We compared the miRNAs between NYHA I-II $(\mathrm{n}=6)$ and NYHA III-IV $(\mathrm{n}=13)$. Interestingly, the elevated miR-5571-5p $(\mathrm{p}<0.05)$ had a significant association with more severe NYHA classification (Fig. 4). In addition, miR-3135b and miR-3908 had no significant difference between NYHA I-II and NYHA III-IV.

\section{Discussion}

As the roles of miRNAs in cardiovascular diseases are increasingly recognized, we aimed to investigate the potential of circulating miRNAs as biomarkers in DCM. Our study identified a novel cluster of plasma miRNAs (miR-3135b, miR-3908, and miR-5571-5p) that was significantly upregulated in DCM patients. In addition, miR-3135b and miR-3908 had the discriminatory power to differentiate between healthy controls and DCM patients nearly similar to the classical NT-proBNP, and miR-5571-5p levels had significant correlation with symptom severity.

Numerous studies have focused on alterations in the levels and compositions of circulating miRNAs in different cardiovascular conditions and pathologies [18, 19]. Upon that, we demonstrated that plasma miR-3135b, miR-3908 and miR-5571-5p might be candidate biomarkers for DCM for the first time. Fan et al. [20] identified miR-423-5p as a biomarker for HF caused by DCM. However, we did not find miR-423-5p levels in DCM to differ from those in the controls. The stage of disease (acute, chronic, treated or untreated), co-morbidity, selected controls and internal references may underlie some of the lack of concordance between these reports. Furthermore, the ROC analysis showed that miR-3135b and miR-5571-5p have similar diagnostic powers for DCM as NT-proBNP. Thus, miRNA signatures in plasma can potentially be developed into biomarkers for HF in the future and could act as complements to NT-proBNP in some special situations.

The stability of circulating miRNAs raises the intriguing speculation that they are taken up by distant cells to regulate their gene expression. Currently, the potential function of extracellular miRNAs is being studied intensively, and some studies have confirmed that circulating miRNAs may indeed function in cell-to-cell communication [21, 22]. Therefore, the differential expression of miRNAs in plasma in DCM might have its pathophysiological effect and might be the new therapeutic target. Moreover, we discovered that plasma miRNA signature was not analogous to the cardiac miRNA expression pattern, providing new sight into the source and release method of circulating miRNAs.

Improvement in symptoms of HF patients caused by DCM is one of the two major goals of treatment. The symptomatic severity was evaluated by questionnaire on health-related quality of life, 6-min walk test, NYHA classification, NT-proBNP concentration, and other criteria. However, some of the aforementioned methods could be hampered by many factors, including educational background, depression, and co-morbidity (such as chronic obstructive pulmonary disease or osteoarthritis), or could have some limitations in particular situations, including unstable angina pectoris and acute myocardial infarction. NT-proBNP was associated with a wide variety of cardiac and non-cardiac causes [23-26], and some patients with advanced HF had normal NT-proBNP levels or had falsely low NT-proBNP levels. Our study showed the expression levels of miR-5571-5p were in line with 
the NYHA classification. Thus, miR-5571-5p might be an objective biomarker for symptomatic severity and might be complementary to pre-existing evaluation methods. Furthermore, the clinically guided up-titration strategy was reported to be suboptimal in achieving target doses of pharmacological agents in HF, which limited the therapeutic effects of the evidence-based HF medications [27-29]. In addition, some meta-analyses concluded that NT-proBNP-guided therapy reduced all-cause mortality in patients with HF compared with usual clinical care [30-32]. Thus, plasma miR-5571-5p might be a candidate biomarker to monitor the disease progression and guide reasonable therapy.

To investigate the physiological functions of the dysregulated miRNAs in response to DCM, the potential targets of the 3 miRNAs were predicted, sequentially, with the commonly used miRNA target prediction software program - TargetScan (http://www.targetscan.org/). We found 322 putative targets for miR-3135b and 314 putative targets for miR-3908; miR-5571-5p was not in the TargetScan database. The downregulated genes (targets of the upregulated miRNAs) were successfully annotated with gene ontology analysis (http://geneontology. org/). It was easily observed that target genes of miR-3135b and miR-3908 were associated with HF-related biological processes, especially the regulation of cardiac muscle contraction, regulation of calcium ion transport, cardiac cell differentiation, negative regulation of cellular process, regulation of metabolic process, regulation of body fluid levels and cardiac myofibril assembly. All of these imply that the 3 miRNAs play important roles in HF. Such assessment could increase the confidence in the potential use of these circulating miRNAs as potential biomarkers for DCM. Mpreover, it is also a step toward further functional studies of these miRNAs.

\section{Limitations of the study}

There are several limitations to this study. One of the major limitations of this study is the sample size, as the results need to be confirmed in a larger population. Secondly, we recruited clinically stable patients who had not received acute treatment for some period of time, and we cannot be certain regarding possible interference of chronic medication. Finally, the precise sources of circulating miRNAs remain largely unknown.

\section{Conclusions}

In summary, we reported that the expression levels of miR-3135b, miR-3908, and miR-5571-5p in plasma might be used as biomarkers for DCM, and increased miR-5571-5p expression levels were found to be associated with a poor NYHA classification. Our work demonstrates that they are promising candidates for this challenge.

\section{Acknowledgements}

We thank the patients and healthy volunteers who participated in this study with great enthusiasm, the doctors and nurses for their assistance with this study.

Sources of funding: This work was supported by grants from the National Natural Science Foundation of China (No. 2012ZX09303-008-002).

\section{Conflict of interest: None declared}

\section{References}

1. Taylor DO, Edwards LB, Boucek MM et al. Registry of the International Society for Heart and Lung Transplantation: twentyfourth official adult heart transplant report — 2007. J Heart Lung Transplant, 2007; 26: 769-781.

2. Ambros V. microRNAs: Tiny regulators with great potential. Cell, 2001; 107: 823-826.

3. Bartel DP. MicroRNAs: Genomics, biogenesis, mechanism, and function. Cell, 2004; 116: 281-297.

4. Eulalio A, Huntzinger E, Izaurralde E. Getting to the root of miRNA-mediated gene silencing. Cell, 2008; 132: 9-14.

5. Filipowicz W, Bhattacharyya SN, Sonenberg N. Mechanisms of post-transcriptional regulation by microRNAs: are the answers in sight?. Nat Rev Genet, 2008; 9: 102-114.

6. Thum T, Catalucci D, Bauersachs J. MicroRNAs: novel regulators in cardiac development and disease. Cardiovasc Res, 2008; 79: 562-570.

7. Van Rooij E, Olson EN. MicroRNAs: Powerful new regulators of heart disease and provocative therapeutic targets. J Clin Invest, 2007; 117: 2369-2376.

8. Eisenberg I, Eran A, Nishino I et al. Distinctive patterns of microRNA expression in primary muscular disorders. Proc Natl Acad Sci USA, 2007; 104: 17016-1721.

9. Latronico M V, Condorelli G. MicroRNAs and cardiac pathology. Nat Rev Cardiol, 2009; 6: 419-429.

10. Naga Prasad SV, Duan ZH, Gupta MK et al. Unique microRNA profile in end-stage heart failure indicates alterations in specific cardiovascular signaling networks. J Biol Chem, 2009; 284: 27487-2799.

11. Van Rooij E, Marshall WS, Olson EN. Toward microRNA-based therapeutics for heart disease: The sense in antisense. Circ Res, 2008; 103: 919-928.

12. Chen JF, Murchison EP, Tang R et al. Targeted deletion of Dicer in the heart leads to dilated cardiomyopathy and heart failure. Proc Natl Acad Sci USA, 2008; 105: 2111-2116.

13. Rao PK, Toyama Y, Chiang HR et al. Loss of cardiac microRNAmediated regulation leads to dilated cardiomyopathy and heart failure. Circ Res, 2009; 105: 585-594.

14. Ikeda S, Kong SW, Lu J et al. Altered microRNA expression in human heart disease. Physiol Genomics, 2007; 31: 367-373. 
15. Voellenkle C, Van Rooij J, Cappuzzello C et al. MicroRNA signatures in peripheral blood mononuclear cells of chronic heart failure patients. Physiol Genomics, 2010; 42: 420-426.

16. Etheridge A, Lee I, Hood L et al. Extracellular microRNA: a new source of biomarkers. Mutat Res, 2011; 717: 85-90.

17. Li JY, Yong TY, Michael MZ et al. Review: The role of microRNAs in kidney disease. Nephrology (Carlton), 2010; 15: 599-608.

18. Xu J, Zhao J, Evan G et al. Circulating microRNAs: novel biomarkers for cardiovascular diseases. J Mol Med (Berl), 2011; 90: 865-875.

19. Laterza O , Lim L, Garrett-Engele PW et al. Plasma MicroRNAs as sensitive and specific biomarkers of tissue injury. Clin Chem, 2009; 55: 1977-1983.

20. Fan KL, Zhang HF, Shen J et al. Circulating microRNAs levels in Chinese heart failure patients caused by dilated cardiomyopathy. Indian Heart J, 2013; 65: 12-16.

21. Xiao C, Calado D P, Galler G et al. MiR-150 controls B cell differentiation by targeting the transcription factor c-Myb. Cell, 2007; 131: 146-159.

22. Zhang Y, Liu D, Chen X et al. Secreted monocytic miR-150 enhances targeted endothelial cell migration. Mol Cell, 2010; 39: 133-144.

23. Anwaruddin S, Lloyd-Jones DM, Baggish A et al. Renal function, congestive heart failure, and amino-terminal pro-brain natriuretic peptide measurement: Results from the ProBNP Investigation of Dyspnea in the Emergency Department (PRIDE) Study. J Am Coll Cardiol, 2006; 47: 91-97.

24. Chang AY, Abdullah SM, Jain T et al. Associations among androgens, estrogens, and natriuretic peptides in young women:
Observations from the Dallas Heart Study. J Am Coll Cardiol, 2007; 49: 109-116.

25. Redfield MM, Rodeheffer RJ, Jacobsen SJ et al. Plasma brain natriuretic peptide concentration: Impact of age and gender. J Am Coll Cardiol, 2002; 40: 976-982.

26. Wang TJ, Larson MG, Levy D et al. Impact of age and sex on plasma natriuretic peptide levels in healthy adults. Am J Cardiol, 2002; 90: 254-258.

27. Calvin JE, Shanbhag S, Avery E et al. Adherence to evidencebased guidelines for heart failure in physicians and their patients: Lessons from the Heart Failure Adherence Retention Trial (HART). Congestive Heart Failure, 2012; 18: 73-78.

28. Komajda M, Lapuerta P, Hermans $\mathrm{N}$ et al. Adherence to guidelines is a predictor of outcome in chronic heart failure: The MAHLER survey. Eur Heart J, 2005; 26: 1653-1659.

29. Troughton RW, Richards AM, Yandle TG et al. The effects of medications on circulating levels of cardiac natriuretic peptides. Ann Med, 2007; 39: 242-260.

30. De Vecchis R, Esposito C, Di Biase G et al. B-type natriuretic peptide-guided versus symptom-guided therapy in outpatients with chronic heart failure: a systematic review with meta-analysis. J Cardiovasc Med, 2014; 15: 122-134.

31. Pufulete M, Higgins JP, Rogers CA et al. Protocol for a systematic review and individual participant data meta-analysis of B-type natriuretic peptide-guided therapy for heart failure. Systematic Rev, 2014; 3: 41.

32. Xin W, Lin Z, Mi S. Does B-type natriuretic peptide-guided therapy improve outcomes in patients with chronic heart failure? A systematic review and meta-analysis of randomized controlled trials. Heart Failure Rev, 2015; 20: 69-80. 